\title{
Анализ параметров GaN-HEMT до и после гамма-нейтронного воздействия
}

\author{
(C) Е.А. Тарасова, С.В. Оболенский, О.Е. Галкин, А.В. Хананова, А.Б. Макаров \\ Нижегородский государственный университет им. Н.И. Лобачевского, \\ 603600 Нижний Новгород, Россия \\ E-mail: thelen@yandex.ru
}

(Получена 27 апреля 2017 г. Принята к печати 12 мая 2017 г.)

Предложен метод математической обработки результатов измерений вольт-фарадных характеристик HEMT $\mathrm{AlGaN} / \mathrm{GaN}$ до и после $\gamma$-нейтронного облучения с флюенсом $0.4 \cdot 10^{14} \mathrm{~cm}^{-2}$. Описаны результаты физико-топологического моделирования HEMT AlGaN/GaN на подложке $\mathrm{SiC}$. Определена погрешность расчета параметров GaN-HEMT, обусловленная погрешностью вычисления профиля распределения электронов.

DOI: 10.21883/FTP.2017.11.45108.22

\section{1. Введение}

Для решения задачи проектирования радиационностойких монолитных интегральных схем (МИС) на основе $\mathrm{GaN}$ необходимо проведение численного моделирования с высокой точностью. Необходимым является расчет выходных вольт-амперных характеристик (BAX) транзисторов, $y$-параметров, коэффициента усиления и пр. Исходными данными для численных расчетов электрофизических параметров являются толщины и уровни легирования полупроводниковых слоев, информация о распределении и подвижности электронов [1,2]. В отличие от тестовых структур, современных HEMT (high electron mobility transistors) емкости изменяются в диапазоне 1.5-5пф, и различие вольт-фарадных характеристик (BФХ) такого типа приборов до и после $\gamma$-нейтроного облучения флюенсом $10^{13}-10^{15} \mathrm{~cm}^{-2}$ и дозой $10^{5}-10^{7}$ рад не превышает $0.01-0.1$ п, что затрудняет проведение анализа радиационно-стимулированного изменения параметров транзисторов. Также открытым остается вопрос об обработке результатов измерений, так как стандартный дифференциальный анализ вольт-фарадных характеристик диодов $\mathrm{AlGaN} / \mathrm{GaN}$ имеет погрешность, связанную с ложным смещением профилей распределения электронов [3]. Поэтому требуется разработка дополнительного метода численного анализа, позволяющего верифицировать полученные из измерений данные.

В работе предложено использовать метод численной обработки ВФХ, комбинирующий методы Тихонова 1-го или 2-го порядка и сглаживание полученной зависимости рациональной функцией.

\section{2. Постановка задачи}

На рис. 1 приведено схематическое изображение исследуемого HEMT AlGaN/GaN. Анализировалось изменение профиля распределения электронов в слое двумерного электронного газа (2DEG) до и после $\gamma$-нейтроного облучения с флюенсом $0.4 \cdot 10^{14} \mathrm{~cm}^{-2}$.
Обозначим $F(u)$ вольт-фарадную характеристику транзистора, полученную с помощью методики, описанной в [3]. Пусть $F(u)=F d_{0}(u)-\mathrm{B} Ф Х$ до облучения, $F(u)=F p_{0}(u)-$ ВФХ после $\gamma$-нейтронного облучения транзистора флюенсом $0.4 \cdot 10^{14} \mathrm{~cm}^{-2}$. Следует отметить, что значения $F\left(u_{i}\right)$ известны для некоторого конечного набора напряжений $a=u_{0}, u_{1}, \ldots, u_{r}=b$. При этом величины $F\left(u_{i}\right)$ измерены не точно, а с некоторой погрешностью. Поэтому задача заключается в расчете значений $F\left(u_{i}\right)$ и построения профиля распределения концентрации электронов, т.е. зависимости величины $N(u)=k_{N} F^{3}(u) / F^{\prime}(u)$ от величины $d(u)=k_{d} / F(u)$, где $k_{N}$ и $k_{d}$ - некоторые заданные числовые коэффициенты.

\section{3. Алгоритмы и результаты решения задачи}

Основной вопрос, возникающий при решении этой задачи, состоит в том, как вычислить производную $F^{\prime}(u)$ от функции, заданной приближенно лишь в конечном наборе точек. В работе был выбран алгоритм, в котором метод наилучших рациональных приближений комбинируется с методом регуляризации Тихонова 1-го или 2-го порядка.

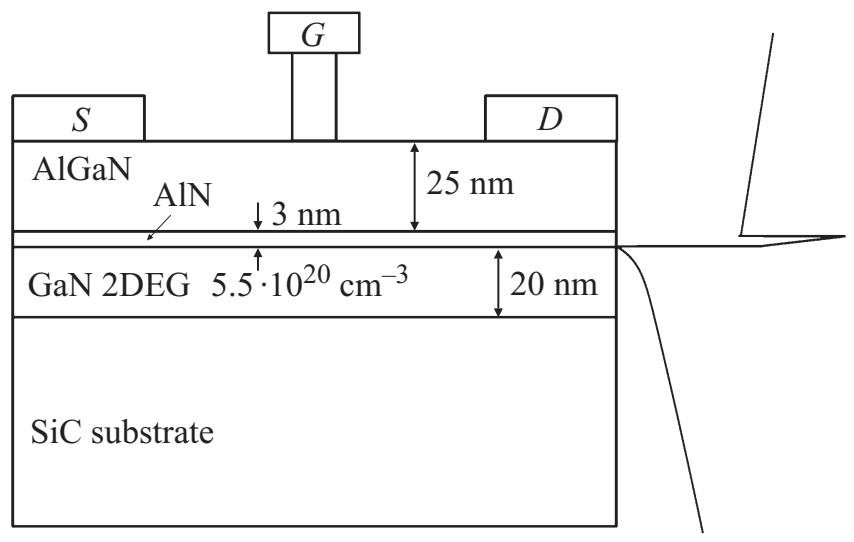

Рис. 1. Структура исследуемого GaN-HEMT и зонная диаграмма подзатворного слоя. $S$ - исток, $D-$ сток, $G-$ затвор. 


\section{4. Описание алгоритма}

Алгоритм состоит из 6 шагов.

1) Задаются целые неотрицательные числа $m$ и $n$.

2) Заданная функция $F(u)$ сглаживается рациональными функциями $R=P / Q$, т. е. отношением многочленов $P$ и $Q$ некоторых степеней $m, n$ соответственно. В частности, при $n=0$ рациональная функция $R=P / Q$ сама является многочленом. Приближение выполняется методом наименьших квадратов, т.е. коэффициенты многочленов $P(u)=a_{0}+a_{1} u+\ldots+a_{m} u^{m}$ и $Q(u)=b_{0}+b_{1} u+\ldots+b_{n} u^{n}$ подбираются так, чтобы минимизировать по $a_{0}, a_{1}, \ldots a_{m}$ и $b_{0}, b_{1}, \ldots, b_{n}$ величину отклонения:

$$
\begin{aligned}
{\left[R\left(u_{1}\right)\right.} & \left.-F\left(u_{1}\right)\right]^{2}+\ldots+\left[R\left(u_{r}\right)=F\left(u_{r}\right)\right]^{2} \\
& =\sum_{k=0}^{r}\left[\frac{a_{0}+a_{1} u_{k}+\ldots+a_{m} u_{k}^{m}}{b_{0}+b_{1} u_{k}+\ldots+b_{n} u_{k}^{n}}-F\left(u_{k}\right)\right]^{2} .
\end{aligned}
$$

Для минимизации (1) применяются: методы Ньютона, градиентного спуска, а также случайный поиск [4]. Далее, используя найденное рациональное приближение $R=P / Q$, рассчитываем и строим профиль распределения электронов по формулам $N_{R}(u)=k_{N} R^{3}(u) / R^{\prime}(u)$ и $d_{R}(u)=k_{d} / R(u)$.

3) В точках $u_{0}, u_{1}, \ldots, u_{r}$ вычисляется разность $G(u)$ исходной функции $F(u)$ и сглаженной функции $R(u)$ : $\delta F\left(u_{k}\right)=F\left(u_{k}\right)-R\left(u_{k}\right), k=1, \ldots, r$.

4) Функцию $\delta F(u)$ сглаживаем методом Тихонова, заодно находя производную $g(u)$ сглаженной функции $G(u)[5,6]$. В методе Тихонова сглаженная функция $G(u)$ имеет вид $G(u)=\int_{a}^{u} g(x) d x+C$, где функция $g(u)$ и константа $C$ подбираются так, чтобы минимизировать функционал (2):

$$
\begin{aligned}
& \int_{a}^{b}\left[\int_{a}^{u} g(x) d x+C-F(u)\right]^{2} d u \\
& \quad+\int_{a}^{b}\left[\alpha g^{2}(u)+\beta g^{\prime 2}(u)+\gamma g^{\prime \prime 2}(u)\right] d u .
\end{aligned}
$$

При этом значения неотрицательных параметров $\alpha, \beta$ и $\gamma$ должны быть достаточно малы и выбираются из некоторых дополнительных соображений. Для минимизации функционала (2) можно использовать соответствующее уравнение Эйлера. При $\alpha>0, \beta>0, \gamma=0$ получим метод Тихонова 1-го порядка, при $\alpha>0, \beta=0$, $\gamma>0$ получим метод Тихонова 2-го порядка.

5) Вычисляется приближение $\Phi(u)$ для исходной функции $F(u)$ и приближение $\Phi^{\prime}(u)$ для производной $F^{\prime}(u): \Phi(u)=R(u)+G(u) ; \Phi^{\prime}(u)=R^{\prime}(u)+g(u)$. Далее проводится вычисление сглаженных профилей по формулам $N_{\Phi}(u)=k_{N} \Phi^{3}(u) / \Phi^{\prime}(u)$ и $d_{\Phi}(u)=k_{d} / \Phi^{\prime}(u)$.

6) Если профили $N_{\Phi}(u)$ и $d_{\Phi}(u)$, построенные с применением метода Тихонова, слабо отличаются от профилей

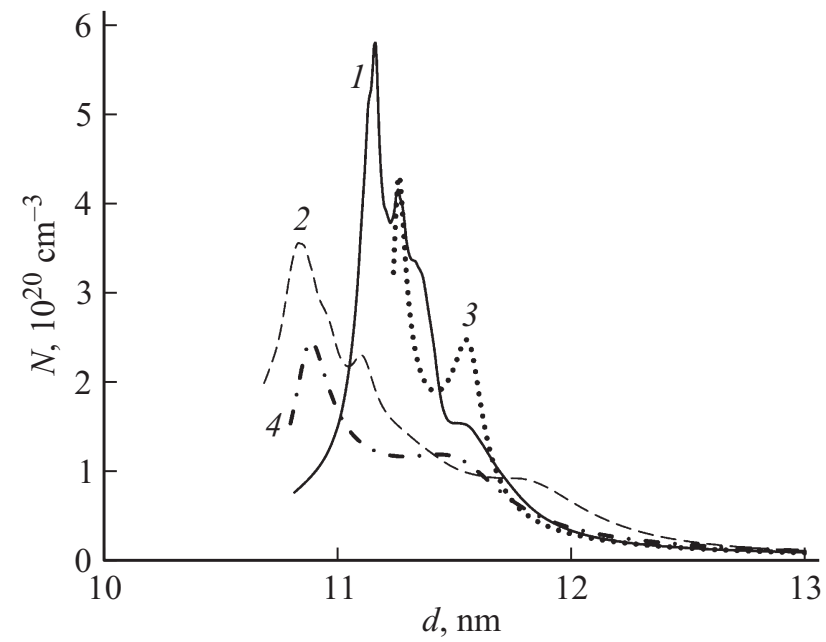

Рис. 2. Результаты расчетов профилей распределения электронов $N$ в GaN-HEMT до (1) и после (2) облучения нейтронами флюенсом $0.4 \cdot 10^{14} \mathrm{~cm}^{-2}$ с использованием рационального приближения и метода Тихонова 1-го порядка, а также полученные стандартным методом [3] до облучения (3) и после облучения (4).

$N_{R}(u)$ и $d_{R}(u)$, соответствующих рациональному приближению $R(u)$, считается, что эти профили $N_{\Phi}(u)$ и $d_{\Phi}(u)$ являются реальными профилями. В противном случае повторяется весь алгоритм действий 1)-6) с другими парами степеней $m, n$ до выполнения итогового условия.

Данный подход к обработке графика функции позволил полностью восстановить профиль распределения электронов в исследуемой гетероструктуре (рис. 2). Помимо определения положения максимума профиля [3] был также восстановлен подлегирующий слой. Наблюдается характерное смещение профиля распределения электронов после нейтронного облучения, аналогичное описанному в [3].

\section{5. Моделирование}

Использование математической обработки результатов измерений крайне важно при оценке радиационной стойкости приборов, так как концентрация электронов в канале НЕМТ определяет значение тока стока транзистора.

Был проведен расчет выходных вольт-амперных характеристик GaN-HEMT на участке насыщения для разных значений максимума профиля концентрации электронов при фиксированном напряжении затвора. Проведено сравнение результатов расчета с экспериментально полученными ВАХ. Погрешность расчета не превысила $10 \%$, что свидетельствует о корректности определения профиля распределения электронов с помощью предложенной математической обработки. В таблице показано, что при ошибке в значении максимума концентрации $\left(d n_{\max }\right)$ в $3-5 \%$ погрешность в расчете тока может достигнуть 5-10\%, ошибка на 10\% по концентрации, дает 
Оценка погрешности расчета

\begin{tabular}{c|c|c}
\hline$d n_{\max }, \%$ & $d I_{d}, \%$ & $d T_{\max }, \%$ \\
\hline 0 & 0 & 0 \\
3 & 5 & 7 \\
5 & 10 & 12 \\
10 & 20 & 25 \\
20 & 50 & 35
\end{tabular}

погрешность в расчете выходного тока $\left(d I_{d}\right) 10-20 \%$, а погрешность в $20 \%$ может привести к ошибке более чем в 2 раза.

Кроме того, погрешность в оценке значения концентрации электронов может привести к некорректному расчету температуры в кристалле транзистора, что в свою очередь приводит к неправильной оценке уровня стойкости прибора. В таблице приведены данные о процентном изменении максимальной температуры канала транзистора $\left(d T_{\max }\right)$ при различной погрешности определения значения максимума профиля распределения электронов.

Таким образом, большая ошибка при расчете температуры канала может привести и к неправильному анализу радиационной стойкости прибора.

С использованием данных по распределению концентрации электронов, полученных для $\mathrm{GaN}$ в этой работе и для GaAs в [3], был проведен аналитический расчет изменения выходного тока транзистора $I_{d}$ на основе $\mathrm{GaAs}$ и $\mathrm{GaN}$ в зависимости от флюенса нейтронного облучения $F$ с учетом и без учета нагрева канала транзистора (рис. 3).

Как видно из рисунка, нагрев канала в GaAs- и GaN-HEMT приводит к увеличению концентрации носителей заряда. B GaAs-HEMT этот эффект играет положительную роль, так как может частично компенсировать



Рис. 3. Расчет зависимости выходного тока $I_{d} \mathrm{GaN}-$ и GaAs-HEMT от флюенса нейтронов с учетом (штриховая линия) и без учета (сплошная) нагрева канала. потерю тока из-за образования большого количества кластеров и субкластеров радиационных дефектов. Однако разогрев канала не сможет полностью компенсировать влияние облучения. В GaN-HEMT количество образующихся дефектов не так велико [3], поэтому нагрев приводит к еще большему увеличению тока в канале, что в свою очередь приводит к ускорению процесса лавинного пробоя. Таким образом, в GaN-HEMT нагрев канала во время облучения приводит к скорой деградации прибора.

С целью проверки корректности предложенного метода определения профиля электронов было проведено физико-топологическое моделирование параметров GaN-HEMT на подложке $\mathrm{SiC}$, со структурой, аналогичной структуре в работе [7], с учетом температурных эффектов. В работе исследовался GaN-HEMT на основе усовершенствованной гетероструктуры, в которую введен тонкий слой (спейсер) AIN между буферным слоем $\mathrm{GaN}$ и барьерным слоем $\mathrm{AlGaN}$ (рис. 1). Влияние такой модификации гетероструктуры было рассмотрено в работе [8]. Сильный поляризационный эффект от введения барьерной прослойки $\mathrm{AlN}$, который имеет бо́льшую ширину запрещенной зоны по сравнению с $\mathrm{AlGaN}$, толщиной не более $3.5 \mathrm{HM}$, приводит к возрастанию концентрации электронов $2 \mathrm{DEG}$, до $n_{s}=3.6 \cdot 10^{13} \mathrm{~cm}^{-2}$, и значительному повышению подвижности электронов $\mu_{e}$, до $2200 \mathrm{~cm}^{2} /(\mathrm{B} \cdot \mathrm{c})$, в силу того что уменьшается вероятность проникновения электронов в $\mathrm{AlGaN}$ [2]. Благодаря новой конструкции была получена максимальная выходная удельная мощность 8.6 Вт/мм на частоте 40 ГГц при длине затвора 150-180 нм, максимальная частота работы прибора составила 120 ГГц [7].

Однако, несмотря на указанные преимущества, одним из важных недостатков GaN-HEMT является большой саморазогрев протекающим током. Большой ток в канале приводит к росту температуры кристаллической решетки на $100 \mathrm{~K}$ и более, что в свою очередь приводит к уменьшению ширины запрещенной зоны и подвижности электронов почти в 10 раз. Уменьшение подвижности электронов приводит к уменьшению выходного тока транзистора с ростом рабочего напряжения [9] и к появлению отрицательного наклона в области насыщения зависимости тока стока от напряжения на нем. Расчет выходных $\mathrm{BAX} I_{d}\left(U_{d}\right)$ GaN-HEMT с учетом тепловых эффектов приведен на рис. 4. Указанные эффекты являются причиной уменьшения выходной мощности транзистора.

Величина саморазогрева главным образом зависит от материала подложки транзистора [11]. В[11] показано, что транзисторы на подложке $\mathrm{SiC}$ нагреваются значительно меньше, чем на сапфировой подложке. В результате расчетов получено, что сапфир, несмотря на то что его теплопроводность меньше на порядок теплопроводности $\mathrm{SiC}(0.3$ против $5 \mathrm{BT} / \mathrm{cm} \cdot \mathrm{K})$, имеет ряд преимушеств в плане дешевизны и простоты технологии выращивания полупроводниковых структур на нем в сравнении с SiC. Альтернативным методом по устранению воздействия саморазогрева на характеристики GaN-HEMT на сапфировой подложке является 




Pис. 4. Стоковые характеристики GaN-HEMT при разных потенциалах на затворе $U_{g}$ : сплошные кривые - данная работа, точки - работа [10].

технология flip-chip [12], при которой структура транзистора монтируется в материал с большой теплопроводностью, такой как $\mathrm{AlN}$, теплопроводность которого равна $\sim 1.8 \mathrm{BT} / \mathrm{cm} \cdot \mathrm{K}$.

В результате расчетов получено, что технология flipchip уменьшает разогрев устройства (максимальная температура в канале $540 \mathrm{~K}$ ) по сравнению с обычным транзистором на сапфировой подложке (максимальная температура в канале $800 \mathrm{~K}$ ). Тем не менее транзистор на подложке $\mathrm{SiC}$ разогревается меньше всего (максимальная температура в канале $404 \mathrm{~K}$ ). Таким образом, корректный расчет температуры при оценке влияния технологии flip-chip также возможен только при использовании описанного в работе подхода к оценке распределения концентрации электронов.

\section{6. Заключение}

Предложенный метод обработки вольт-фарадных характеристик современных НЕМТ, измеренных с низкой погрешностью, комбинирующий метод рациональных приближений и методы регуляризации Тихонова 1-го и 2-го порядков, позволил восстановить профили распределения электронов в канале GaN-HEMT до и после $\gamma$-нейтронного облучения с флюенсом $0.4 \cdot 10^{14} \mathrm{~cm}^{-2}$. Сравнение расчетных и экспериментальных характеристик свидетельствует об адекватности данного подходка к обработке экспериментальных ВФХ.

С использованием полученных результатов проведен расчет передаточных характеристик GaAs- и GaN-HEMT при нейтронном облучении с различным уровнем флюенса, с учетом и без учета нагрева канала транзистора. Учет температурных эффектов позволил определить уровни радиационной стойкости приборов.

В результате проведенного физико-топологического моделирования HEMT AlGaN/GaN выявлены преимущества использования технологии flip-chip для уменьшения процесса саморазогрева транзистора.
Работа выполнена при поддержке грантами РФФИ № 14-02-0058, 15-02-07935, 15-47-02294-р_поволжье_а, МОН РФ (соглашение от 27.09.2013 г. № 02.В.49.21.003) и государственным заданием МОН РФ (код проекта 2183).

\section{Список литературы}

[1] Н.В. Басаргина, И. Ворожцова, С.М. Дубровских, О.В. Ткачев, В.П. Шукайло, Е.А. Тарасова, А.Ю. Чурин, С.В. Оболенский. Вестн. ННГУ им. Н.И. Лобачевского, 1 (3), 61 2013.

[2] Ю.А. Матвеев, Ю.В. Федоров. Нано- и микросистемная техника, № 5, 39 (2011).

[3] Е.А. Тарасова, Е.С. Оболенская, А.В. Хананова, С.В. Оболенский, В.Е. Земляков, В.И. Егоркин, А.В. Неженцев, А.В. Сахаров, А.Ф. Цацульников, В.В. Лундин, Е.Е. Заварин, Г.В. Медведев. ФТП, 50 (12), 1599 (2016).

[4] В.И. Бердышев, Л.В. Петрак. Аппроксимация функций, сжатие численной информации, приложения (Екатеринбург, ИММ УрО РАН, 1999).

[5] А.Н. Тихонов, В.Я. Арсенин. Методы решения некорректных задач (М., Наука, 1979).

[6] Н.С. Бахвалов, Н.П. Жидков, Г.М. Кобельков. Численные методы (М., БИНОМ. Лаборатория знаний, 2008).

[7] Yifeng $\mathrm{Wu}$, Primit Parikh. Intern. Symp. Compound Semiconductor (January/February 2006).

[8] I.P. Smorchkova, L. Chen, T. Mates, L. Shen, S. Heikman, B. Moran, S. Keller, S.P. Den Baars, J.S. Speck, U.K. Mishra. J. Appl. Phys., 90, 5196 (2001).

[9] G. Meneghesso, G. Verzellesi, F. Danesin, F. Rampazzo, F. Zano, A. Tazzoli, M. Meneghini, E. Zanoni. IEEE Trans. Dev. Mater. Reliab., 8 (2), 332 (2008).

[10] Abhitosh Vais. Thesis for Erasmus Mundus Master Programme Nanoscience \& Nanotechnology, (Microwave Electronics Laboratory Department of Microtechnology \& Nanoscience, Chalmers University of Technology, Gothenburg, Sweden, 2012).

[11] G.K. Wachutka. IEEE Trans. Comput.-Aided Des. Integr. Circuits Syst., 9 (11), 1141 (1990).

[12] Jie Sun, H. Fatima, A. Koudymov, A. Chitnis, X. Hua, H.-M. Wang, J. Zhang, G. Simin, J. Yang, M. Asif Khan. IEEE Electron Dev. Lett., 24 (6), 375 (2003).

Редактор Л.В. Шаронова

\section{Analysis of GaN HEMT parameters before and after gamma-neutron impact}

\author{
E.A. Tarasova, S.V. Obolensky., O.E. Galkin, \\ A.V. Hananova, A.B. Makarov \\ Lobachevsky State University of Nizhny Novgorod, \\ 603950 Nizhny Novgorod, Russia
}

Abstract A method for mathematical processing of the results AlGaN/GaN HEMT of volt - capacitance characteristics measurements before and after gamma-neutron irradiation with fluence of $0.4 \cdot 10^{14} \mathrm{~cm}^{-2}$ is proposed. The results of the modeling for AlGaN/GaN HEMT on a SiC substrate are described. The error in calculating GaN HEMT parameters due to the error in calculating the electron distribution profile was observed. 